\title{
AUTOMORPHISMS OF THE DEFORMATION SPACE OF A KLEINIAN GROUP ${ }^{1}$
}

\author{
BY
}

JAMES A. GENTILESCO

\begin{abstract}
In the following paper, we determine the biholomorphic automorphisms of a cross-product of Teichmuller spaces. This in turn helps us to determine the biholomorphic automorphisms of the deformation space of a Kleinian group using the fact that its holomorphic universal covering space is a cross-product of Teichmüller spaces. Among other interesting results, we show that in general the deformation space is not a homogeneous space.
\end{abstract}

Preliminaries. It has been known for some time (see Bers [2]) that the classical modular group of the Fuchsian group $\Gamma$, denoted by $\operatorname{Mod}(\Gamma)$, acts as a group of (biholomorphic) automorphisms of the Teichmüller space $T(\Gamma)$ and that this action is effective except in a few low dimensional cases [2]. We assume throughout that $\Gamma$ is finitely generated of the first kind without elliptic elements. In a deep result, $\mathbf{H}$. L. Royden proved that the (biholomorphic) automorphism group of the Teichmüller space $T(\Gamma)$, where $U / \Gamma$ is a compact Riemann surface of genus $\geqslant 3$, is precisely the action of $\operatorname{Mod}(\Gamma)$, i.e., Aut $T(\Gamma)=\operatorname{Mod}(\Gamma)$, if genus $U / \Gamma \geqslant 3$ [8]. Later C. Earle and I. Kra generalized this result to the case where $U / \Gamma$ is compact with finitely many punctures with the exception of a few cases [5]. The technique, in both cases that of H. L. Royden, is to exploit the fact that a biholomorphic automorphism of $T(\Gamma)$ induces a complex linear isometry between cotangent spaces over corresponding points on $T(\Gamma)$ identified by the automorphism and then to characterize such complex linear isometries in such a way that this correspondence is pointwise (i.e., depending on the point) the action of an element of $\operatorname{Mod}(\Gamma)$ and then to use the proper discontinuity of $\operatorname{Mod}(\Gamma)$ to show that globally (i.e., independent of the point) the automorphism is the action of some element of $\operatorname{Mod}(\Gamma)[8]$.

Received by the editors July 30, 1977 and, in revised form, December 8, 1977

AMS (MOS) subject classifications (1970). Primary 32G15, 32M05; Secondary 32G05, 30A46.

Key words and phrases. Fuchsian group, Kleinian group, Riemann surface, Teichmüller space, deformation space, modular group, Kobayashi metric, Finsler structure.

${ }^{1}$ This paper is based on the author's doctoral dissertation at State University of New York at Stony Brook under the direction of Professor Irwin Kra.

(C) 1979 American Mathematical Society $0002-9947 / 79 / 0000-0060 / \$ 04.50$ 
In the attempt to determine the biholomorphic automorphisms of a crossproduct of Teichmüller spaces and then more generally those of the deformation space of a Kleinian group, I proceeded along the lines mentioned above, i.e., to characterize the mappings induced between the cotangent spaces of a cross-product (which is a direct sum of cotangent spaces of the Teichmüller spaces in the cross-product) at corresponding points identified by an arbitrary biholomorphic automorphism. In order to condense this paper and highlight the main results, one should assume the following results:

[A] There exists a Finsler structure on the tangent bundle to a crossproduct of Teichmüller spaces (which turns out to be the max of the Finsler structures on the tangent bundle to each space) such that the Kobayashi metric is the integrated form of the norm given by this Finsler structure.

[B] Biholomorphic automorphisms of a cross-product of Teichmüller spaces induces complex linear isometries on the tangent bundle in the norm given by the Finsler structure (the max of $L^{\infty}$ norms).

[C] Biholomorphic automorphisms of a cross-product of Teichmüller spaces induce complex linear isometries on the cotangent bundle in the norm dual to that given by the Finsler structure (which turns out to be the sum of $L^{1}$ norms).

Statement $[\mathrm{A}]$ is an application to Teichmüller space of a more general theorem of Royden [9]. Statements [B] and [C] are consequences of statement [A]. Details are supplied in the author's thesis [10].

We remind the reader that a point in Teichmüler space is an equivalence class of Riemann surfaces and that the cotangent space over such a point is the Banach space $Q(X)$ of holomorphic quadratic differentials on any surface $X$ in the equivalence class with finite $L^{1}$ norm ([1] and [4]).

\section{Complex linear isometries between cotangent spaces.}

Definition. Let $x, x^{\prime} \in \bigoplus_{i=1}^{n} B_{i}$, where the $B_{i}$ are Banach spaces, $x=$ $\left(x_{1}, \ldots, x_{n}\right), x^{\prime}=\left(x_{1}^{\prime}, \ldots, x_{n}^{\prime}\right) . x$ is said to be perpendicular to $x^{\prime}$ (written $\left.x \perp x^{\prime}\right)$ if for each $i$, either $x_{i}$ or $x_{i}^{\prime}$ is zero.

LEMMA I. Let $f: Q\left(X_{1}\right) \oplus \cdots \oplus Q\left(X_{n}\right) \rightarrow Q\left(Y_{1}\right) \oplus \cdots \oplus Q\left(Y_{n}\right)$ be a complex linear isometry between direct sums of the complex Banach spaces of holomorphic quadratic differentials on the Riemann surfaces $X_{i}, Y_{i}$ in the norm $\|\cdot\|+\cdots+\|\cdot\|$, where $\|\phi\|=\frac{1}{2} \int|\phi|, \phi$ a holomorphic quadratic differential. Let $x=\left(x_{1}, \ldots, x_{n}\right)$ and $x^{\prime}=\left(x_{1}^{\prime}, \ldots, x_{n}^{\prime}\right)$ be such that $x \perp x^{\prime}$. Let $\left(x_{1}, \ldots, x_{n}\right) \rightarrow^{f}\left(y_{1}, \ldots, y_{n}\right),\left(x_{1}^{\prime}, \ldots, x_{n}^{\prime}\right) \rightarrow^{f}\left(y_{1}^{\prime}, \ldots, y_{n}^{\prime}\right)$. Then for each $i$, $1 \leqslant i \leqslant n$, one of the following holds.

(i) $y_{i}=0$,

(ii) $y_{i}^{\prime}=0$, or

(iii) $y_{i}^{\prime}=r_{i} y_{i}$ for some $r_{i}>0$. 
REMARK. It turns out by Lemma II that case (iii) never occurs.

Proof. Let $x=\left(x_{1}, \ldots, x_{n}\right)$ and $x^{\prime}=\left(x_{1}^{\prime}, \ldots, x_{n}^{\prime}\right)$ be such that $x \perp x^{\prime}$. Let $\left(x_{1}, \ldots, x_{n}\right) \rightarrow^{f}\left(y_{1}, \ldots, y_{n}\right),\left(x_{1}^{\prime}, \ldots, x_{n}^{\prime}\right) \rightarrow^{f}\left(y_{1}^{\prime}, \ldots, y_{n}^{\prime}\right)$. Since $f$ is an isometry $\left\|x_{1}\right\|+\cdots+\left\|x_{n}\right\|=\left\|y_{1}\right\|+\cdots+\left\|y_{n}\right\|$ and $\left\|x_{1}^{\prime}\right\|+\cdots+$ $\left\|x_{n}^{\prime}\right\|=\left\|y_{1}^{\prime}\right\|+\cdots+\left\|y_{n}^{\prime}\right\|$. Since $f$ is linear, $\left(x_{1}+x_{1}^{\prime}, \ldots, x_{n}+x_{n}^{\prime}\right) \rightarrow^{f}\left(y_{1}\right.$ $\left.+y_{1}^{\prime}, \ldots, y_{n}+y_{n}^{\prime}\right)$; hence $\left\|x_{1}+x_{1}^{\prime}\right\|+\cdots+\left\|x_{n}+x_{n}^{\prime}\right\|=\left\|y_{1}+y_{1}^{\prime}\right\|$ $+\cdots+\left\|y_{n}+y_{n}^{\prime}\right\|$. Now $\left\|x_{1}+x_{1}^{\prime}\right\|+\cdots+\left\|x_{n}+x_{n}^{\prime}\right\|=\left\|x_{1}\right\|$ $+\cdots+\left\|x_{n}\right\|+\left\|x_{1}^{\prime}\right\|+\cdots+\left\|x_{n}^{\prime}\right\|$ since $x \perp x^{\prime}$. By substitution $\| y_{1}+$ $y_{1}^{\prime}\|+\cdots+\| y_{n}+y_{n}^{\prime}\|=\| y_{1}\|+\| y_{1}^{\prime}\|+\cdots+\| y_{n}\|+\| y_{n}^{\prime} \|$. Since $\| y_{i}+$ $y_{i}^{\prime}\|\leqslant\| y_{i}\|+\| y_{i}^{\prime} \|$ for norms, all $i$, it follows that $\left\|y_{i}+y_{i}^{\prime}\right\|=\left\|y_{i}\right\|+\left\|y_{i}^{\prime}\right\|$, all $i$. Let $y_{i}=\phi_{i}, y_{i}^{\prime}=\eta_{i}$; then equivalently

$$
\frac{1}{2} \int_{Y_{i}}\left|\phi_{i}+\eta_{i}\right|=\frac{1}{2} \int_{Y_{i}}\left|\phi_{i}\right|+\frac{1}{2} \int_{Y_{i}}\left|\eta_{i}\right|, \quad \text { all } i
$$

or $\int_{Y_{i}}\left|\phi_{i}\right|+\left|\eta_{i}\right|-\left|\phi_{i}+\eta_{i}\right|=0$ which implies that $\left|\phi_{i}+\eta_{i}\right|=\left|\phi_{i}\right|+\left|\eta_{i}\right|$ a.e., since the integrand is nonnegative by the triangle inequality. Now $\left|\phi_{i}+\eta_{i}\right|=$ $\left|\phi_{i}\right|+\left|\eta_{i}\right|$ a.e. implies that $\eta_{i} / \phi_{i}$ can take on a.e. (that is, a.e. in every parametric disk) only values that are nonnegative or $\infty$.

It is then clear, by continuity, that $\left|\phi_{i}+\eta_{i}\right|=\left|\phi_{i}\right|+\left|\eta_{i}\right|$ everywhere and hence $\eta_{i} / \phi_{i}$ is a meromorphic function that is always nonnegative or infinite. Since nonconstant meromorphic functions are open mappings, one of the following must be true:

(i) $\phi_{i}=0$,

(ii) $\eta_{i}=0$, or

(iii) $\eta_{i}=r_{i} \phi_{i}$ for some $r_{i}>0$.

LEMMA II. Let $f: B_{1} \oplus \cdots \oplus B_{n} \rightarrow B_{1}^{\prime} \oplus \cdots \oplus B_{n}^{\prime}$ be a complex linear isometry (where the $B_{i}, B_{i}^{\prime}$ are complex Banach spaces) in the norm $\|\cdot\|$ $+\cdots+\|\cdot\|$, where $\|\cdot\|$ is a norm for each Banach space. Let $x=$ $\left(x_{1}, \ldots, x_{n}\right)$ and $x^{\prime}=\left(x_{1}^{\prime}, \ldots, x_{n}^{\prime}\right)$ be such that $x \perp x^{\prime}$. Let $\left(x_{1}, \ldots, x_{n}\right)$ $\rightarrow^{f}\left(y_{1}, \ldots, y_{n}\right),\left(x_{1}^{\prime}, \ldots, x_{n}^{\prime}\right) \rightarrow^{f}\left(y_{1}^{\prime}, \ldots, y_{n}^{\prime}\right)$ and suppose that $y_{i}=y_{i}^{\prime}$ for some $i$; then $y_{i}=y_{i}^{\prime}=0$.

PRoOF. Let $x=\left(x_{1}, \ldots, x_{n}\right) \rightarrow^{f}\left(y_{1}, \ldots, y_{n}\right), x^{\prime}=\left(x_{1}^{\prime}, \ldots, x_{n}^{\prime}\right)$ $\rightarrow^{f}\left(y_{1}^{\prime}, \ldots, y_{n}^{\prime}\right)$ be such that $x \perp x^{\prime}$ and suppose $y_{i}=y_{i}^{\prime}$ for some $i$. Since $f$ is an isometry $\left\|x_{1}\right\|+\cdots+\left\|x_{n}\right\|=\left\|y_{1}\right\|+\cdots+\left\|y_{n}\right\|$ and $\left\|x_{1}^{\prime}\right\|$ $+\cdots+\left\|x_{n}^{\prime}\right\|=\left\|y_{1}^{\prime}\right\|+\cdots+\left\|y_{n}^{\prime}\right\|$. Since $f$ is linear $\left(x_{1}-x_{1}^{\prime}, \ldots, x_{n}-\right.$ $\left.x_{n}^{\prime}\right) \rightarrow^{f}\left(y_{1}-y_{1}^{\prime}, \ldots, y_{n}-y_{n}^{\prime}\right)$ and therefore $\left\|x_{1}-x_{1}^{\prime}\right\|+\cdots+\left\|x_{n}-x_{n}^{\prime}\right\|$ $=\left\|y_{1}-y_{1}^{\prime}\right\|+\cdots+\left\|y_{n}-y_{n}^{\prime}\right\|$. The term $\left\|y_{i}-y_{i}^{\prime}\right\|=0$ by hypothesis. Now $\left\|x_{1}-x_{1}^{\prime}\right\|+\cdots+\left\|x_{n}-x_{n}^{\prime}\right\|=\left\|x_{1}\right\|+\cdots+\left\|x_{n}\right\|=\left\|x_{1}^{\prime}\right\|$ $+\cdots+\left\|x_{n}^{\prime}\right\|$ since $x \perp x^{\prime}$. 
By substitution,

$$
\begin{aligned}
\left\|y_{1}-y_{1}^{\prime}\right\|+\cdots & +\left\|y_{i-1}-y_{i-1}^{\prime}\right\|+\left\|y_{i+1}, \ldots, y_{i+1}^{\prime}\right\|+\cdots+\left\|y_{n}-y_{n}^{\prime}\right\| \\
= & \left\|y_{1}\right\|+\cdots+\left\|y_{n}\right\|+\left\|y_{1}^{\prime}\right\|+\cdots+\left\|y_{n}^{\prime}\right\| \\
& =2\left\|y_{i}\right\|+\left(\left\|y_{1}\right\|+\left\|y_{1}^{\prime}\right\|+\cdots+\left\|y_{i-1}\right\|+\left\|y_{i-1}^{\prime}\right\|\right. \\
& \left.\quad+\left\|y_{i+1}\right\|+\left\|y_{i+1}^{\prime}\right\|+\cdots+\left\|y_{n}\right\|+\left\|y_{n}^{\prime}\right\|\right) .
\end{aligned}
$$

Since by the triangle inequality, $\left\|y_{j}-y_{j}^{\prime}\right\| \leqslant\left\|y_{j}\right\|+\left\|y_{j}^{\prime}\right\|$ all $j$, it follows that $\left\|y_{i}\right\|=0$, thus $y_{i}=0$. Hence $y_{i}=y_{i}^{\prime}=0$.

TheOREM I. Let $f: Q\left(X_{1}\right) \oplus \cdots \oplus Q\left(X_{n}\right) \rightarrow Q\left(Y_{1}\right) \oplus \cdots \oplus Q\left(Y_{n}\right)$ be a complex linear isometry between direct sums of the complex Banach spaces of holomorphic quadratic differentials on the Riemann surfaces $X_{i}, Y_{i}$, in the norm $\|\cdot\|+\cdots+\|\cdot\|$, where $\|\phi\|=\frac{1}{2} \int|\phi|, \phi$ a holomorphic quadratic differential. Then there exists a permutation $\sigma \in S_{n}$ (symmetric group on $n$ letters), such that

$$
f_{\mid Q\left(X_{i}\right)}: Q\left(X_{i}\right) \stackrel{\text { onto }}{\rightarrow} Q\left(Y_{o(i)}\right), \quad 1 \leqslant i \leqslant n .
$$

Proof. The proof is by induction on $n$.

$n=1$. $f: Q\left(X_{1}\right) \rightarrow Q\left(Y_{1}\right)$. There is nothing to prove. Assume that the theorem is true for $n=k-1$. We must prove that the theorem is true for $n=k$, that is, for $f: Q\left(X_{1}\right) \oplus \cdots \oplus Q\left(X_{k}\right) \rightarrow Q\left(Y_{1}\right) \oplus \cdots \oplus Q\left(Y_{k}\right)$. Let $0 \neq\left(x_{1}, 0, \ldots, 0\right) \rightarrow^{f}\left(y_{1}, \ldots, y_{k}\right)$ be fixed and $\left(0, x_{2}^{\prime}, \ldots, x_{k}^{\prime}\right)$ $\rightarrow^{f}\left(y_{1}^{\prime}, \ldots, y_{k}^{\prime}\right)$ be arbitrary. (Note that if $Q\left(X_{1}\right)=\{0\}$, we cannot pick an $x_{1} \neq 0$. In that case, choose any point $\left(0, \ldots, x_{i}, 0, \ldots, 0\right) \in \bigoplus_{i} Q\left(X_{i}\right)$, $x_{i} \neq 0$; otherwise $\bigoplus_{i} Q\left(X_{i}\right)=\{0\}$ and there is nothing to prove. Let ( $x_{1}^{\prime}$, $\left.x_{2}^{\prime}, \ldots, 0, x_{i+1}^{\prime}, \ldots, x_{k}^{\prime}\right)$ be the other arbitrary point.)

Since $x_{1} \neq 0$ and $f$ is an isometry, then there exists a $y_{i} \neq 0$. By a permutation, say $y_{1} \neq 0$, then by Lemma I either $y_{1}^{\prime}=0$ or $y_{1}^{\prime}=r_{1} y_{1}, r_{1}>0$. If $y_{1}^{\prime}=r_{1} y_{1}, r_{1}>0$, then by linearity,

$$
\left(0, x_{2}^{\prime} / r_{1}, \ldots, x_{n}^{\prime} / r_{1}\right) \stackrel{f}{\rightarrow}\left(y_{1}, y_{2}^{\prime} / r_{1}, \ldots, y_{k}^{\prime} / r_{1}\right)
$$

so that by Lemma II, $y_{1}=0$, which is a contradiction. Hence $y_{1}^{\prime}=0$. Since $\left(0, x_{2}^{\prime}, \ldots, x_{n}^{\prime}\right)$ is arbitrary, we see that $f:\left(0, x_{2}^{\prime}, \ldots, x_{k}^{\prime}\right) \rightarrow\left(0, y_{2}^{\prime}, \ldots, y_{k}^{\prime}\right)$, that is $f_{\text {|rest }}: Q\left(X_{2}\right) \oplus \cdots \oplus Q\left(X_{k}\right) \rightarrow Q\left(Y_{2}\right) \oplus \cdots \oplus Q\left(Y_{k}\right)$. We claim this mapping is onto, for if not, then there exist points $\left(x_{1}, x_{2}, \ldots, x_{k}\right)$, $x_{1} \neq 0,\left(0, y_{2}, \ldots, y_{k}\right)$ such that $f\left(x_{1}, x_{2}, \ldots, x_{k}\right)=\left(0, y_{2}, \ldots, y_{k}\right)$. Since $f$ is onto and we have already assumed $Q\left(Y_{1}\right) \neq\{0\}$, then there exists points $\left(x_{1}^{\prime}, \ldots, x_{k}^{\prime}\right) \in \bigoplus_{i} Q\left(X_{i}\right), \quad\left(y_{1}, 0, \ldots, 0\right) \in \bigoplus_{i} Q\left(Y_{i}\right), y_{1} \neq 0$, such that $f\left(x_{1}^{\prime}, \ldots, x_{k}^{\prime}\right)=\left(y_{1}, 0, \ldots, 0\right)$. Since $\left(0, y_{2}, \ldots, y_{k}\right) \perp\left(y_{1}, 0, \ldots, 0\right)$, we can apply Lemma I to $f^{-1}$ and get that $x_{1}^{\prime}=0$ or $x_{1}^{\prime}=r_{1} x_{1}$ since $x_{1} \neq 0$. If $x_{1}^{\prime}=r_{1} x_{1}$, then $f^{-1}\left(y_{1} / r_{1}, 0, \ldots, 0\right)=\left(x_{1}, x_{2}^{\prime} / r_{1}, \ldots, x_{k}^{\prime} / r_{1}\right)$ and thus by Lemma II applied to $f^{-1}, x_{1}=0$ a contradiction. Hence $x_{1}^{\prime}=0$ and thus $f(0$, 
$\left.x_{2}^{\prime}, \ldots, x_{k}^{\prime}\right)=\left(y_{1}, 0, \ldots, 0\right)$. Since $y_{1} \neq 0$ this, however, contradicts the above. Hence $f_{\text {|rest }}$ is onto. Now by induction, there exists a $\tau \in S_{k-1}$ such that $f_{\mid Q\left(X_{i}\right)}: Q\left(X_{i}\right) \rightarrow^{\text {onto }} Q\left(Y_{\tau(i)}\right), 2 \leqslant i \leqslant k$. We claim that $f_{\mid Q\left(X_{1}\right)}: Q\left(X_{1}\right)$ $\rightarrow^{\text {onto }} Q\left(Y_{1}\right)$ and hence the theorem, since there would exist $\sigma \in S_{k}$ such that $f_{\mid Q\left(X_{i}\right)}: Q\left(X_{i}\right) \rightarrow^{\text {onto }} Q\left(Y_{\sigma(i)}\right), 1 \leqslant i \leqslant k$.

For let $\left(x_{1}, \ldots, x_{k}\right)$ be arbitrary and let $x_{1}$ vary while $x_{2}, \ldots, x_{k}$ are fixed. By linearity, $f\left(x_{1}, \ldots, x_{k}\right)=f\left(x_{1}, 0, \ldots, 0\right)+f\left(0, x_{2}, \ldots, x_{k}\right)$. It is clear that $f\left(0, x_{2}, \ldots, x_{k}\right)$ remains invariant as $x_{1}$ is allowed to vary. We will show that $f\left(x_{1}, 0, \ldots, 0\right)=\left(y_{1}, 0, \ldots, 0\right)$ and hence it follows that $f\left(x_{1}, \ldots, x_{k}\right)$ only varies in the first component of the image direct sum as $x_{1}$ is allowed to vary which implies the claim. So suppose there exists some $y_{i} \neq 0,2 \leqslant i \leqslant k$. Then since $f_{\text {|rest }}$ is onto, there exists a point $\left(0, x_{2}^{\prime}, \ldots, x_{k}^{\prime}\right)$ such that $f(0$, $\left.x_{2}^{\prime}, \ldots, x_{k}^{\prime}\right)=\left(0, \ldots, y_{i}, 0, \ldots, 0\right)$. But then by Lemma II, $y_{i}=0$, a contradiction. Thus $f\left(x_{1}, 0, \ldots, 0\right)=\left(y_{1}, 0, \ldots, 0\right)$.

THEOREM II. Let $f: Q\left(X_{1}\right) \oplus \cdots \oplus Q\left(X_{m}\right) \rightarrow Q\left(Y_{1}\right) \oplus \cdots \oplus Q\left(Y_{n}\right)$ where $m>n$ and $f$ and the $Q\left(X_{i}\right), Q\left(Y_{i}\right)$ are as in the previous theorem. Then at least $m-n$ of the $Q\left(X_{i}\right)$ are $\{0\}$, and if we eliminate $m-n$ trivial spaces from $\bigoplus_{i=1}^{m} Q\left(X_{i}\right)$, and possibly renumber the remaining spaces, then $f: Q\left(X_{1}\right)$ $\oplus \cdots \oplus Q\left(X_{n}\right) \rightarrow^{\text {onto }} Q\left(Y_{1}\right) \oplus \cdots \oplus Q\left(Y_{n}\right)$ and splits componentwise up to a permutation (as in the previous theorem).

Proof. Add $m-n$ trivial spaces $\{0\}$ onto $\bigoplus_{i=1}^{n} Q\left(Y_{i}\right)$. Then $f: Q\left(X_{1}\right)$ $\oplus \cdots \oplus Q\left(X_{m}\right) \rightarrow Q\left(Y_{1}\right) \oplus \cdots \oplus Q\left(Y_{n}\right) \oplus\{0\} \oplus \cdots \oplus\{0\}$. Since $f$ is a complex linear and we now have the same number of components in each direct sum, the previous theorem implies that there exists a $\sigma \in S_{m}$ such that $f_{\mid Q\left(X_{i}\right)}: Q\left(X_{i}\right) \rightarrow^{\text {onto }} Q\left(Y_{\sigma(i)}\right), 1 \leqslant i \leqslant m$. Since we have added on $m-n$ trivial spaces, there must exist at least $m-n$ trivial spaces among $\bigoplus_{i=1}^{m} Q\left(X_{i}\right)$. Eliminate precisely $m-n$ of them. Then after a possible renumbering of the remaining spaces, we have $f: Q\left(X_{1}\right) \oplus \cdots \oplus Q\left(X_{n}\right) \rightarrow$ $Q\left(Y_{1}\right) \oplus \cdots \oplus Q\left(Y_{n}\right)$ and the previous theorem implies the rest.

REMARK. The previous section has the following geometrical interpretation:

Definition. A unit vector $x$ is called an extreme point of the unit ball if and only if there do not exist linearly independent vectors $x^{\prime}$ and $x^{\prime \prime}$. with $x^{\prime}+x^{\prime \prime}=x$ and $\left\|x^{\prime}\right\|+\left\|x^{\prime \prime}\right\|=\|x\|=1$

Using the proof of Lemma I, one can prove:

LEMma. The unit vector $x=\left(x_{1}, \ldots, x_{n}\right)$ in $Q\left(X_{1}\right) \oplus \cdots \oplus Q\left(X_{n}\right)$ is an extreme point of the unit ball if and only if there is some $i$ such that $x_{j}=0$, if $i \neq j$.

Using the fact that complex linear isometries carry extreme points into 
extreme points, one can also prove Theorem I directly using the above lemma.

2. The modular group of a cross-product. Let $T\left(\Gamma_{1}\right) \times \cdots \times T\left(\Gamma_{n}\right)$ be a cross-product of Teichmüller spaces of Fuchsian groups $\Gamma_{i}, 1 \leqslant i \leqslant n$, each finitely generated of the first kind without elliptic elements. We define the modular group of this cross-product to be $\operatorname{Mod}\left(\Gamma_{1}\right) \times \cdots \times \operatorname{Mod}\left(\Gamma_{n}\right)$ where $\operatorname{Mod}\left(\Gamma_{i}\right)$ is the modular group of $T\left(\Gamma_{i}\right), i \leqslant i \leqslant n[5]$. Let $x=\left(x_{1}, \ldots, x_{n}\right)$ $\in T\left(\Gamma_{1}\right) \times \cdots \times T\left(\Gamma_{n}\right)$ and $\theta=\left(\theta_{1}, \ldots, \theta_{n}\right) \in \operatorname{Mod}\left(\Gamma_{1}\right) \times \cdots \times$ $\operatorname{Mod}\left(\Gamma_{n}\right)$. Then $\theta(x)=\left(\theta_{1}\left(x_{1}\right), \ldots, \theta_{n}\left(x_{n}\right)\right) \in T\left(\Gamma_{1}\right) \times \cdots \times T\left(\Gamma_{n}\right)$.

Proposition I [10]. The modular group acts on the cross-product $T\left(\Gamma_{1}\right)$ $\times \cdots \times T\left(\Gamma_{n}\right)$ :

(i) as a group of biholomorphic automorphisms,

(ii) as a group of isometries in the Kobayashi metric,

(iii) as a properly discontinuous group,

(iv) effectively, if type $\Gamma_{i}=\left(g_{i}, n_{i}\right)$, i.e., $U / \Gamma_{i}$ is compact of genus $g_{i}$ with $n_{i}$ punctures, and $2 g_{i}+n_{i}>4$, all $i$.

Corollary. If type $\Gamma_{i}=\left(g_{i}, n_{i}\right)$ satisfies $2 g_{i}+n_{i}>4$, all $i$, then statements (i) and (iv) from the above proposition imply that

$$
\operatorname{Mod}\left(\Gamma_{1}\right) \times \cdots \times \operatorname{Mod}\left(\Gamma_{n}\right) \subseteq \operatorname{Aut} T\left(\Gamma_{1}\right) \times \cdots \times T\left(\Gamma_{n}\right) .
$$

LeMMA III [10]. Let $M$ be a complex analytic manifold. Let $G$ be a properly discontinuous group of biholomorphic self-mappings of $M$. Let $\theta$ be any biholomorphic self-mapping of $M$. If for each $x \in M$ there exists an element $\gamma_{x} \in G$ such that $\theta(x)=\gamma_{x}(x)$, then $\theta \in G$.

\section{Automorphisms of cross-products.}

THEOREM III. Suppose $\Gamma_{1}, \ldots, \Gamma_{n}$ are Fuchsian groups which have no elliptic elements and the type $\Gamma_{i}=\left(g_{i}, n_{i}\right)$ satisfies $2 g_{i}+n_{i}>4$, all $i$ (i.e., $\Gamma_{i}$ is nonexceptional). Then Aut $T\left(\Gamma_{1}\right) \times \cdots \times T\left(\Gamma_{n}\right)$ is the semidirect product of $\operatorname{Mod}\left(\Gamma_{1}\right) \times \cdots \times \operatorname{Mod}\left(\Gamma_{n}\right)$ by the finite subgroup $H$, where $H$ is defined as follows: Let $N=\left\{(i, j) \mid 1 \leqslant i<j \leqslant n\right.$ and type $\Gamma_{i}=$ type $\left.\Gamma_{j}\right\}$. For each $(i, j) \in N$ define $f_{i j}: T\left(\Gamma_{i}\right) \rightarrow T\left(\Gamma_{j}\right)$ to be a chosen biholomorphic mapping (such mappings exist between Teichmüller spaces of groups of the same type [3]). For each $(i, j) \in N$, let $h_{i j}=\left(k_{1}, \ldots, k_{n}\right)$, where $k_{r}=$ id if $r \neq i, j, k_{i}=f_{i j}$, and $k_{j}=f_{i j}^{-1}$. Then $H$ is the subgroup generated by the elements $h_{i j}$.

Lemma IV. Let the product $\operatorname{Mod}\left(\Gamma_{1}\right) \times \cdots \times \operatorname{Mod}\left(\Gamma_{n}\right)$ by $H$ (as above) be the set of all elements of the form $g \circ h$, where $g \in \operatorname{Mod}\left(\Gamma_{1}\right) \times \cdots \times$ $\operatorname{Mod}\left(\Gamma_{n}\right)$ and $h \in H$. Then the product $\operatorname{Mod}\left(\Gamma_{1}\right) \times \cdots \times \operatorname{Mod}\left(\Gamma_{n}\right)$ by $H$ is $a$ group under composition and operates properly discontinuously on $T\left(\Gamma_{1}\right)$ $\times \cdots \times T\left(\Gamma_{n}\right)$. 
Proof. Clearly the identity element belongs to the set and the associative property holds. We need to show that every element has an inverse and the closure property. We first remark that if $g \in \operatorname{Mod}\left(\Gamma_{1}\right) \times \cdots \times \operatorname{Mod}\left(\Gamma_{n}\right)$ and $h \in H$ are arbitrary, then $h \circ g \circ h^{-1} \in \operatorname{Mod}\left(\Gamma_{1}\right) \times \cdots \times \operatorname{Mod}\left(\Gamma_{n}\right)$ since $h$ permutes the factors of $T\left(\Gamma_{1}\right) \times \cdots \times T\left(\Gamma_{n}\right), g \in$ Aut $T\left(\Gamma_{1}\right)$ $\times \cdots \times$ Aut $T\left(\Gamma_{n}\right)$; hence $h \circ g \circ h^{-1} \in$ Aut $T\left(\Gamma_{1}\right) \times \cdots \times$ Aut $T\left(\Gamma_{n}\right)$ $=\operatorname{Mod}\left(\Gamma_{1}\right) \times \cdots \times \operatorname{Mod}\left(\Gamma_{n}\right)$ (since Aut $T\left(\Gamma_{i}\right)=\operatorname{Mod}\left(\Gamma_{i}\right)$; Royden [8]). Thus if $g_{1} \circ h_{1}$ and $g_{2} \circ h_{2}$ are any two elements, $\left(g_{1} \circ h_{1}\right) \circ\left(g_{2} \circ h_{2}\right)=$ $\left(h_{1} \circ g_{3}\right) \circ\left(g_{2} \circ h_{2}\right)=\left(h_{1} \circ g_{4}\right) \circ h_{2}=\left(g_{5} \circ h_{1}\right) \circ h_{2}=g_{5} \circ h_{3}$, where the $g_{i}$ $\in \operatorname{Mod}\left(\Gamma_{1}\right) \times \cdots \times \operatorname{Mod}\left(\Gamma_{n}\right)$ and the $h_{i} \in H$. If $g \circ h$ belongs to the product, then let $(g \circ h)^{-1}=\left(h^{-1} \circ g^{-1} \circ h\right) \circ h^{-1}$, and $\left(h^{-1} \circ g^{-1} \circ h\right) \circ h^{-1}$ belongs to the product by the above remark.

By definition, the group $H$ is clearly finite; hence $\operatorname{Mod}\left(\Gamma_{1}\right) \times \cdots \times$ $\operatorname{Mod}\left(\Gamma_{n}\right)$ is of finite index in the product $\operatorname{group} \operatorname{Mod}\left(\Gamma_{1}\right) \times \cdots \times \operatorname{Mod}\left(\Gamma_{n}\right)$ by $H$ and since $\operatorname{Mod}\left(\Gamma_{1}\right) \times \cdots \times \operatorname{Mod}\left(\Gamma_{n}\right)$ operates properly discontinuously on $T\left(\Gamma_{1}\right) \times \cdots \times T\left(\Gamma_{n}\right)$, it follows that the product $\operatorname{Mod}\left(\Gamma_{1}\right)$ $\times \cdots \times \operatorname{Mod}\left(\Gamma_{n}\right)$ by $H$ operates properly discontinuously on $T\left(\Gamma_{1}\right)$ $\times \cdots \times T\left(\Gamma_{n}\right)$.

Proof (OF THEOREM). From the corollary at the end of the last section and the definition of $H$, it is clear that the product $\operatorname{Mod}\left(\Gamma_{1}\right) \times \cdots \times \operatorname{Mod}\left(\Gamma_{n}\right)$ by $H$ is contained in Aut $T\left(\Gamma_{1}\right) \times \cdots \times T\left(\Gamma_{n}\right)$. Hence it suffices to prove containment in the other direction.

Let $h \in$ Aut $T\left(\Gamma_{1}\right) \times \cdots \times T\left(\Gamma_{n}\right)$. Since the Kobayashi metric on $T\left(\Gamma_{1}\right)$ $\times \cdots \times T\left(\Gamma_{n}\right)$ is induced by a Finsler structure on the tangent space invariant under biholomorphic mappings (see [A]), $h$ induces a complex linear isometry between the tangent spaces (in the max norm given by the Finsler structure on the cross-product, see [B]) over each pair of points $x$ and $h(x)$ in $T\left(\Gamma_{1}\right) \times \cdots \times T\left(\Gamma_{n}\right)$. Since the cotangent space is dual to the tangent space with dual norm, the induced mapping $h^{*}$ between cotangent spaces over $x$ and $h(x)$ is a complex linear isometry in the dual norm, which by a previous theorem is the sum of $L^{1}$ norms (see [C]). Hence by a previous theorem (see §1) $h^{*}$ splits componentwise up to a permutation, that is, $h_{Q\left(X_{i}\right)}^{*}: Q\left(X_{i}\right) \rightarrow$ $Q\left(Y_{\sigma(i)}\right)$ for some $\sigma \in S_{n}$. Since $\Gamma_{1}, \ldots, \Gamma_{n}$ are assumed to be of nonexceptional types, this implies by a theorem of Earle and Kra [5] that $\Gamma_{i}$ and $\Gamma_{\sigma(i)}$ are of the same type $\left(g_{i}, n_{i}\right)$. Hence by definition of $H$, there exists an element $g \in H$ such that $g_{\mid T\left(\Gamma_{i}\right)}: T\left(\Gamma_{i}\right) \rightarrow T\left(\Gamma_{\sigma(i)}\right)$ all $i$, and hence the induced mapping $g_{\mid Q\left(Y_{o(i)}^{\prime}\right)}: Q\left(Y_{\sigma(i)}^{\prime}\right) \rightarrow Q\left(X_{i}^{\prime}\right)$ all $i$. Then one has that $(h \circ g)_{Q Q\left(X_{i}\right)}^{*}$ : $Q\left(X_{i}\right) \rightarrow Q\left(X_{i}^{\prime}\right)$ all $i$. By the results of Royden, generalization of Earle and Kra [5], $X_{i}$ is conformally equivalent to $X_{i}^{\prime}$, for all $i$. This implies the existence of $\Psi_{i} \in \operatorname{Mod}\left(\Gamma_{i}\right)$ such that $h \circ g(x)=\left(\Psi_{1}\left(x_{1}\right), \ldots, \Psi_{n}\left(x_{n}\right)\right)$, where $x=$ $\left(x_{1}, \ldots, x_{n}\right) \in T\left(\Gamma_{1}\right) \times \cdots \times T\left(\Gamma_{n}\right)$ [4], or $h(x)=\Psi \circ g^{-1}(x), \quad \Psi \in$ 
$\operatorname{Mod}\left(\Gamma_{1}\right) \times \cdots \times \operatorname{Mod}\left(\Gamma_{n}\right), g^{-1} \in H$. Since $x \in T\left(\Gamma_{1}\right) \times \cdots \times T\left(\Gamma_{n}\right)$ is arbitrary, there exists an element $\theta \in\left(\operatorname{Mod}\left(\Gamma_{1}\right) \times \cdots \times \operatorname{Mod}\left(\Gamma_{n}\right)\right) H$ such that $h(x)=\theta(x)$ for all $x$ (see above lemma and §2). Hence $h \in$ Aut $T\left(\Gamma_{1}\right)$ $\times \cdots \times T\left(\Gamma_{n}\right)$ implies that $h \in\left(\operatorname{Mod}\left(\Gamma_{1}\right) \times \cdots \times \operatorname{Mod}\left(\Gamma_{n}\right)\right) H$.

We only have left to show that $\operatorname{Mod}\left(\Gamma_{1}\right) \times \cdots \times \operatorname{Mod}\left(\Gamma_{n}\right)$ is a normal subgroup of Aut $T\left(\Gamma_{1}\right) \times \cdots \times T\left(\Gamma_{n}\right)$. It suffices to show $f\left(\operatorname{Mod}\left(\Gamma_{1}\right)\right.$ $\left.\times \cdots \times \operatorname{Mod}\left(\Gamma_{n}\right)\right) f^{-1} \subseteq \operatorname{Mod}\left(\Gamma_{1}\right) \times \cdots \times \operatorname{Mod}\left(\Gamma_{n}\right)$, because by using inverses, $f\left(\operatorname{Mod}\left(\Gamma_{1}\right) \times \cdots \times \operatorname{Mod}\left(\Gamma_{n}\right)\right) f^{-1} \supseteq \operatorname{Mod}\left(\Gamma_{1}\right) \times \cdots \times \operatorname{Mod}\left(\Gamma_{n}\right)$ as well and hence the claim. Let $f \in$ Aut $T\left(\Gamma_{1}\right) \times \cdots \times T\left(\Gamma_{1}\right)$ and $g \in$ $\operatorname{Mod}\left(\Gamma_{1}\right) \times \cdots \times \operatorname{Mod}\left(\Gamma_{n}\right)$. If $f \in H$, then $f \circ g \circ f^{-1}$ maps each component $T\left(\Gamma_{i}\right)$ onto itself. Thus $f \circ g \circ f^{-1} \in \operatorname{Mod}\left(\Gamma_{1}\right) \times \cdots \times \operatorname{Mod}\left(\Gamma_{n}\right)$ (since Aut $T\left(\Gamma_{i}\right)=\operatorname{Mod}\left(\Gamma_{i}\right)$; Royden [8]). Then if $f \in$ Aut $T\left(\Gamma_{1}\right) \times \cdots \times$ $T\left(\Gamma_{n}\right)$ is arbitrary, then $f=f_{2} \circ f_{1}$ where $f_{1} \in H, f_{2} \in \operatorname{Mod}\left(\Gamma_{1}\right) \times \cdots \times$ $\operatorname{Mod}\left(\Gamma_{n}\right)$ and $f \circ g \circ f^{-1}=f_{2} \circ\left(f_{1} \circ g \circ f_{1}^{-1}\right) \circ f_{2}^{-1}=f_{2} \circ g_{1} \circ f_{2}^{-1} \in$ $\operatorname{Mod}\left(\Gamma_{1}\right) \times \cdots \times \operatorname{Mod}\left(\Gamma_{n}\right)$ since $g_{1}, f_{2} \in \operatorname{Mod}\left(\Gamma_{1}\right) \times \cdots \times \operatorname{Mod}\left(\Gamma_{n}\right)$. By definition of $H$, clearly $\left(\operatorname{Mod}\left(\Gamma_{1}\right) \times \cdots \times \operatorname{Mod}\left(\Gamma_{n}\right)\right) \cap H=\{$ id $\}$. Hence Aut $T\left(\Gamma_{1}\right) \times \cdots \times T\left(\Gamma_{n}\right)$ is the semidirect product of $\operatorname{Mod}\left(\Gamma_{1}\right) \times \cdots \times$ $\operatorname{Mod}\left(\Gamma_{n}\right)$ by $H$.

THEOREM IV. Suppose $\Gamma_{1}, \ldots, \Gamma_{n}$ are Fuchsian groups which have no elliptic elements and type $\Gamma_{i}$ is not $(1,1)$ or $(0,4)$, all $i$. Then Aut $T\left(\Gamma_{1}\right) \times \cdots \times$ $T\left(\Gamma_{n}\right)$ is the semidirect product of Aut $T\left(\Gamma_{1}\right) \times \cdots \times$ Aut $T\left(\Gamma_{n}\right)$ by the finite subgroup $\tilde{H}$, where $\tilde{H}$ is defined as follows: Let $\tilde{N}=\{(i, j) \mid 1<i<j<n$ and $\exists f_{i j}: T\left(\Gamma_{i}\right) \rightarrow T\left(\Gamma_{j}\right)$ biholomorphic $\}$. For each $(i, j) \in \tilde{N}$, let $h_{i j}=\left(k_{1}, \ldots, k_{n}\right)$, where $k_{r}=$ id if $r \neq i, j, k_{i}=f_{i j}$, and $k_{j}=f_{i j}^{-1}$. Then $\tilde{H}$ is the subgroup generated by the $h_{i j}$.

PRoof. Clearly the semidirect product of Aut $T\left(\Gamma_{1}\right) \times \cdots \times$ Aut $T\left(\Gamma_{n}\right)$ by $\tilde{H}$ is contained in Aut $T\left(\Gamma_{1}\right) \times \cdots \times T\left(\Gamma_{n}\right)$. Hence we need to prove only containment in the other direction. We remark that if type $\left(\Gamma_{i}\right)=(0,3)$ for some $i$, then $T\left(\Gamma_{i}\right)=$ pt. since $\operatorname{dim}_{C} T\left(\Gamma_{i}\right)=0$. Thus Aut $T\left(\Gamma_{i}\right)=\{$ id $\}$ and neither affects nor contributes anything to the result.

If type $\left(\Gamma_{i}\right)$ is exceptional, not $(0,3)$, and by hypothesis not $(1,1)$ or $(0,4)$, then there exists a Fuchsian group $\tilde{\Gamma}_{i}$ of nonexceptional type such that $T\left(\tilde{\Gamma}_{i}\right)$ is biholomorphically equivalent to $T\left(\Gamma_{i}\right)$ [3]. It is then obvious that one can replace the original cross-product $T\left(\Gamma_{1}\right) \times \cdots \times T\left(\Gamma_{n}\right)$ by the biholomorphically equivalent cross-product $T\left(\tilde{\Gamma}_{1}\right) \times \cdots \times T\left(\tilde{\Gamma}_{n}\right)$, where $\Gamma_{i}$ $=\tilde{\Gamma}_{i}$ if type $\Gamma_{i}$ is nonexceptional or $(0,3)$, via a biholomorphic mapping $f$ : $T\left(\Gamma_{1}\right) \times \cdots \times T\left(\Gamma_{n}\right) \rightarrow T\left(\tilde{\Gamma}_{1}\right) \times \cdots \times T\left(\tilde{\Gamma}_{n}\right)$ which splits componentwise, that is $f_{\mid T\left(\Gamma_{i}\right)}: T\left(\Gamma_{i}\right) \rightarrow T\left(\tilde{\Gamma}_{i}\right), 1 \leqslant i \leqslant n$. Let $h \in$ Aut $T\left(\Gamma_{1}\right) \times \cdots \times$ $T\left(\Gamma_{n}\right)$. Then $g=f \circ h \circ f^{-1}: T\left(\tilde{\Gamma}_{1}\right) \times \cdots \times T\left(\tilde{\Gamma}_{n}\right) \rightarrow T\left(\tilde{\Gamma}_{1}\right) \times \cdots \times$ $T\left(\tilde{\Gamma}_{n}\right)$ is biholomorphic, and by the previous theorem and the triviality of 
type $(0,3)$ (if it appears) $g$ splits componentwise up to a permutation, that is, there exists a $\sigma \in S_{n}$ such that $g_{\mid T\left(\tilde{\Gamma}_{i}\right)}: T\left(\tilde{\Gamma}_{i}\right) \rightarrow T\left(\tilde{\Gamma}_{\sigma(i)}\right)$. Since $f$ splits componentwise, this implies that $h_{\mid T\left(\Gamma_{i}\right)}: T\left(\Gamma_{i}\right) \rightarrow T\left(\Gamma_{\sigma(i)}\right)$. Since $h$ evidently permutes the factors, and $\tilde{H}$ by definition is a group of transpositions of the factors, there exists $g \in \tilde{H}$ such that $\theta=h \circ g^{-1} \in$ Aut $T\left(\Gamma_{1}\right) \times \cdots \times$ Aut $T\left(\Gamma_{n}\right)$. Hence $h=\theta \circ g$ where $\theta \in$ Aut $T\left(\Gamma_{1}\right) \times \cdots \times$ Aut $T\left(\Gamma_{n}\right)$ and $g \in \tilde{H}$.

Analogous to the previous theorem, Aut $T\left(\Gamma_{1}\right) \times \cdots \times$ Aut $T\left(\Gamma_{n}\right)$ is a normal subgroup of Aut $T\left(\Gamma_{1}\right) \times \cdots \times T\left(\Gamma_{n}\right)$. Clearly Aut $T\left(\Gamma_{1}\right)$ $\times \cdots \times$ Aut $T\left(\Gamma_{n}\right) \cap \tilde{H}=\{$ id $\}$. Hence Aut $T\left(\Gamma_{1}\right) \times \cdots \times T\left(\Gamma_{n}\right)$ is the semidirect product of Aut $T\left(\Gamma_{1}\right) \times \cdots \times$ Aut $T\left(\Gamma_{n}\right)$ by $\tilde{H}$.

\section{Biholomorphic mappings between cross-products.}

THEOREM V. Let $h: T\left(\Gamma_{1}\right) \times \cdots \times T\left(\Gamma_{m}\right) \rightarrow T\left(\tilde{\Gamma}_{1}\right) \times \cdots \times T\left(\tilde{\Gamma}_{n}\right), m \geqslant$ $n$, be a biholomorphic mapping between cross-products of Teichmüller spaces. Then at least $m-n$ of the $T\left(\Gamma_{i}\right)$ are trivial, and if we eliminate $m-n$ trivial spaces and possibly renumber the remaining spaces, then $h: T\left(\Gamma_{1}\right) \times \cdots \times$ $T\left(\Gamma_{n}\right) \rightarrow T\left(\tilde{\Gamma}_{1}\right) \times \cdots \times T\left(\tilde{\Gamma}_{n}\right)$.

Proof. Let $h: T\left(\Gamma_{1}\right) \times \cdots \times T\left(\Gamma_{m}\right) \rightarrow T\left(\tilde{\Gamma}_{1}\right) \times \cdots \times T\left(\tilde{\Gamma}_{n}\right), m \geqslant n$, be biholomorphic. Then for each pair of points $x=\left(x_{1}, \ldots, x_{m}\right) \in T\left(\Gamma_{1}\right)$ $\times \cdots \times T\left(\Gamma_{m}\right)$ and $h(x) \in T\left(\tilde{\Gamma}_{1}\right) \times \cdots \times T\left(\tilde{\Gamma}_{n}\right)$ there is an induced complex linear isometry $f: Q\left(X_{1}\right) \oplus \cdots \oplus Q\left(X_{m}\right) \rightarrow Q\left(Y_{1}\right) \oplus \cdots \oplus$ $Q\left(Y_{n}\right)$ on the corresponding cotangent spaces over $x$ and $h(x)$. If $m>n$, then there exist at least $m-n Q\left(X_{i}\right)=\{0\}$ by a previous theorem (see $\S 1$ ). Since $\operatorname{dim}_{C} T\left(\Gamma_{i}\right)=\operatorname{dim}_{C} Q\left(X_{i}\right)$, the result follows. By eliminating $m-n$ trivial spaces and possibly renumbering the remaining spaces, then $h: T\left(\Gamma_{1}\right)$ $\times \cdots \times T\left(\Gamma_{n}\right) \rightarrow T\left(\tilde{\Gamma}_{1}\right) \times \cdots \times T\left(\tilde{\Gamma}_{n}\right)$. If $m=n$, the statement holds vacuously.

COROllaRY I. There does not exist a biholomorphic mapping between an $n$-fold product of Teichmüller spaces and an m-fold product, unless the number of nontrivial spaces in each product is the same. If the number of nontrivial spaces is the same and in addition we assume all nontrivial types nonexceptional in both cross-products, then a biholomorphic mapping exists if and only if the nontrivial types in one product are precisely those appearing among the nontrivial types of the other (counting multiplicities).

REMARK. Exceptional types, if any, must appear in a prescribed manner.

Proof. The first statement follows directly from the theorem. Assume that the number of nontrivial spaces in each product are the same and each nontrivial type is also nonexceptional in both cross-products. If the nontrivial types appearing in one product are precisely those appearing in the other 
(counting multiplicities), clearly there exists a biholomorphic mapping between products, since Teichmüller spaces of the same type are biholomorphically equivalent [3].

Conversely, if $h$ is biholomorphic under the above assumptions, the induced mapping on cotangent spaces over corresponding points splits componentwise up to a permutation (see $\S 1$ ), and using the result of Earle and $\mathrm{Kra}$ [5], the nontrivial types in one product of Teichmüller spaces are precisely those in the other product (counting multiplicities).

COROllary II. A cross-product of nontrivial Teichmüller spaces is never a Teichmüller space.

Proof. Follows directly from theorem.

Corollary III. If $h: T\left(\Gamma_{1}\right) \times \cdots \times T\left(\Gamma_{n}\right) \rightarrow T\left(\tilde{\Gamma}_{1}\right) \times \cdots \times T\left(\tilde{\Gamma}_{n}\right)$ is biholomorphic and $\Gamma_{i}, \tilde{\Gamma}_{i}$ are nonexceptional, then $h$ splits componentwise up to a permutation, that is, there exists a $\sigma \in S_{n}$ such that $h_{\mid T\left(\Gamma_{i}\right)}: T\left(\Gamma_{i}\right)$ $\rightarrow^{\text {onto }} T\left(\Gamma_{\sigma(i)}\right)$, all $i$.

Proof. By the above theorem, $\exists \sigma \in S_{n}$ such that type $\Gamma_{i}=$ type $\tilde{\Gamma}_{\sigma(i)}$. Since Teichmüller spaces of the same type are biholomorphically equivalent, there exists $f: T\left(\tilde{\Gamma}_{1}\right) \times \cdots \times T\left(\tilde{\Gamma}_{n}\right) \rightarrow T\left(\Gamma_{1}\right) \times \cdots \times T\left(\Gamma_{n}\right)$ biholomorphic, $f$ splitting componentwise up to a permutation. Then $f \circ h$ : $T\left(\Gamma_{1}\right) \times \cdots \times T\left(\Gamma_{n}\right) \rightarrow T\left(\Gamma_{1}\right) \times \cdots \times T\left(\Gamma_{n}\right)$, so that by a previous theorem (see $\S 3$ ), $f \circ h$ belongs to the semidirect product of $\operatorname{Mod}\left(\Gamma_{1}\right)$ $\times \cdots \times \operatorname{Mod}\left(\Gamma_{n}\right)$ by $H$. Therefore, $f \circ h$ splits componentwise up to a permutation, and hence $h$ splits componentwise up to a permutation.

5. Automorphisms of the deformation space. Let $G$ be a finitely generated nonelementary Kleinian group with region of discontinuity $\Omega$. Let $f$ be a quasi-conformal automorphism of $C \cup\{\infty\} . f$ is said to be compatible with the group $G$ if $f G f^{-1}$ is again a Kleinian group and $f$ is conformal on $\Lambda(G)$, the limit set of $G$. (It is not known if this latter condition is automatically satisfied by all finitely generated Kleinian groups.) $f$ is called normalized if $f$ fixes 0,1 , and $\infty$. Two normalized compatible quasi-conformal mappings $f_{1}$, $f_{2}$ are called equivalent if $f_{1}(z)=f_{2}(z)$ for all $z \in \Lambda(G)$. Each equivalence class is a point in $T(G, \Omega)$, the deformation space of $G$. Let $\Delta_{1}, \ldots, \Delta_{n}$ be a maximal collection of nonequivalent connected components of $\Omega$, i.e., there does not exist $g \in G$ such that $g\left(\Delta_{i}\right)=\Delta_{j}$, for $i \neq j$. Let $G_{\Delta_{1}}, \ldots, G_{\Delta_{n}}$ be the corresponding stability subgroups, i.e., $G_{\Delta_{i}}=\left\{g \in G / g\left(\Delta_{i}\right)=\Delta_{i}\right\}$. Then $T(G, \Omega) \cong T\left(G_{\Delta_{1}}, \Delta_{1}\right) \times \cdots \times T\left(G_{\Delta_{n}}, \Delta_{n}\right)$ (biholomorphically), where $T\left(G_{\Delta_{i}}, \Delta_{i}\right)$ is the deformation space of $G$ supported in $\Delta_{i}$ (see [6]).

Let $\Delta$ be a component of $\Omega$ and $G_{\Delta}=\{g \in G / g(\Delta)=\Delta\}$. Since $G$ is nonelementary, there exists a holomorphic universal covering $h: U \rightarrow \Delta(U$ 
the upper half-plane). Let $\Gamma$ be the group of conformal automorphisms of $U$ such that $\gamma \in \Gamma$ if there exists an element $\chi(\gamma) \in G$ such that $h \circ \gamma=$ $\chi(\gamma) \circ h$. There is an exact sequence of Kleinian groups $\{1\} \rightarrow H \hookrightarrow \hookrightarrow^{\text {inj }} \Gamma$ $\rightarrow^{x} G \rightarrow\{1\}$ where $H$ is the covering group of $h: U \rightarrow \Delta$. $\Gamma$ is Fuchsian and $U / \Gamma$ is conformally equivalent to $\Delta / G_{\Delta} . \Gamma$ is called the Fuchsian model of $G$ over $\Delta[6]$.

Definition. We call a Kleinian group $G$ exceptional if for its Fuchsian model $\Gamma_{1}, \ldots, \Gamma_{n}$, some $\Gamma_{i}$ is of exceptional type.

THEOREM VI. Let $G$ be a finitely generated nonelementary, nonexceptional Kleinian group. Let $h: T(G, \Omega) \rightarrow T(G, \Omega)$ be a biholomorphic self-mapping of $T(G, \Omega)$. Then there exists a permutation $\sigma \in S_{n}$ such that $h_{\mid T\left(G_{\Delta_{i}}, \Delta_{i}\right)}$ : $T\left(G_{\Delta_{i}}, \Delta_{i}\right) \rightarrow{ }^{\text {onto }} T\left(G_{\Delta_{o(i)}}, \Delta_{\sigma(i)}\right)$ biholomorphically, $1 \leqslant i \leqslant n$. ( $h$ acts on the product via the isomorphism.)

Proof. The universal covering space of $T(G, \Omega)$ is $T\left(\Gamma_{1}\right) \times \cdots \times T\left(\Gamma_{n}\right)$ for some Fuchsian groups $\Gamma_{1}, \ldots, \Gamma_{n}$ (see [7]). The holomorphic covering mapping is $\pi=\pi_{1} \times \cdots \times \pi_{n}$, where $\pi_{i}: T\left(\Gamma_{i}\right) \rightarrow T\left(G_{\Delta_{i}}, \Delta_{i}\right)$ is the holomorphic covering mapping for each component, $1 \leqslant i \leqslant n$. Let $h$ : $T(G, \Omega) \rightarrow T(G, \Omega)$ be a biholomorphic self-mapping of $T(G, \Omega)$. Let $\tilde{h}$ : $T\left(\Gamma_{1}\right) \times \cdots \times T\left(\Gamma_{n}\right) \rightarrow T\left(\Gamma_{1}\right) \times \cdots \times T\left(\Gamma_{n}\right)$ be a lifting of $h$ to the universal covering space. Then by the theorem on cross-product of Teichmüller spaces (see §3), there exists a permutation $\sigma \in S_{n}$ such that $h_{\mid T\left(\Gamma_{i}\right)}$ : $T\left(\Gamma_{i}\right) \rightarrow^{\text {onto }} T\left(\Gamma_{\sigma(i)}\right)$ biholomorphically, $1 \leqslant i \leqslant n$. Since $\pi$ is of the form $\pi=\pi_{1} \times \cdots \times \pi_{n}, \pi_{i}: T\left(\Gamma_{i}\right) \rightarrow T\left(G_{\Delta_{i}}, \Delta_{i}\right)$ it follows easily that $h_{\mid T\left(G_{\Delta_{i}}, \Delta_{i}\right.}$ : $T\left(G_{\Delta_{i}}, \Delta_{i}\right) \rightarrow^{\text {onto }} T\left(G_{\Delta_{o(i)}}, \Delta_{\sigma(i)}\right)$ biholomorphically. Let

$$
T(G, \Omega)=\frac{T\left(\Gamma_{1}\right) \times \cdots \times T\left(\Gamma_{n}\right)}{\operatorname{Mod}_{H_{1}}\left(\Gamma_{1}\right) \times \cdots \times \operatorname{Mod}_{H_{n}}\left(\Gamma_{n}\right)}
$$

be the deformation space, via the isomorphism (see [6], [7]) of the finitely generated nonelementary Kleinian group $G$. Let

$$
\operatorname{Mod}(G, \Omega)=\frac{\operatorname{Mod}^{H_{1}}\left(\Gamma_{1}\right) \times \cdots \times \operatorname{Mod}^{H_{n}}\left(\Gamma_{n}\right)}{\operatorname{Mod}_{H_{1}}\left(\Gamma_{1}\right) \times \cdots \times \operatorname{Mod}_{H_{n}}\left(\Gamma_{n}\right)}
$$

be the associated modular group acting on $T(G, \Omega)$, where $H_{i}=$ the holomorphic covering group of $h_{i}: U \rightarrow \Delta_{i} ; H_{i} \subset \Gamma_{i} \operatorname{Mod}^{H_{i}}\left(\Gamma_{i}\right)=$ the subgroup of $\operatorname{Mod}\left(\Gamma_{i}\right)$ induced by all quasi-conformal automorphisms $w$ : $U \rightarrow U$ such that $w \Gamma_{i} w^{-1}=\Gamma_{i}$ and $w H_{i} w^{-1}=H_{i} \operatorname{Mod}_{H_{i}}\left(\Gamma_{i}\right)=$ the subgroup of $\operatorname{Mod}^{H_{i}}\left(\Gamma_{i}\right)$ (and $\left.\operatorname{Mod}\left(\Gamma_{i}\right)\right)$ induced by all quasi-conformal automorphisms $w: U \rightarrow U$ such that $w \gamma w^{-1} \gamma^{-1} \in H_{i}$ for all $\gamma \in \Gamma_{i}$ (see [6]). 
THEOREM VII. Let $G$ be a finitely generated nonelementary, nonexceptional Kleinian group with nonequivalent components $\Delta_{1}, \ldots, \Delta_{n}$ and Fuchsian model $\Gamma_{1}, \ldots, \Gamma_{n}$. Assume, in addition, that $G$ does not contain elliptic elements and that $\Delta_{i}$ is simply connected for all $i, 1 \leqslant i \leqslant n$. Then Aut $T(G, \Omega)$ is the semidirect product of $\operatorname{Mod}(G, \Omega)$ by the subgroup $H$, where $H$ is defined as in $\S 3$.

Proof. If $\Delta_{i}$ is simply connected, then $H_{i}=\{$ id $\}$ since $H_{i} \cong \pi_{1}\left(\Delta_{i}\right)$ from which it follows easily that $\operatorname{Mod}_{\{\text {id }\}}\left(\Gamma_{i}\right)=\{$ id $\}$ for $1 \leqslant i \leqslant n$ [6]. Hence $T(G, \Omega)=T\left(\Gamma_{1}\right) \times \cdots \times T\left(\Gamma_{n}\right)$, a cross-product of Teichmüller spaces, where $\Gamma_{1}, \ldots, \Gamma_{n}$ is the Fuchsian model of $G$. Since $G$ does not contain elliptic elements, $\Gamma_{i}$ has no elliptic elements, $1 \leqslant i \leqslant n$. Also $G$ nonexceptional by definition means that each $\Gamma_{i}$ is of nonexceptional type. Hence the theorem on cross-products of Teichmüller spaces (see \$3) yields the result.

REMARK. The requirement that $G$ not contain elliptic elements is necessary. If $G$ contains elliptic elements, then, in general, one has $\operatorname{Mod}(G, \Omega) H \subsetneq$ Aut $T(G, \Omega)$ (properly contained) already. We give a simple example to illustrate. Let $\Gamma$ be a finitely generated Fuchsian group with signature $(2,2 ; 2$, $3)$, that is $U / \Gamma$ is a compact Riemann surface with two distinguished points $p_{1}$ and $p_{2}$ such that if $z_{1}, z_{2} \in U$ are such that $\pi\left(z_{i}\right)=p_{i}, i=1,2$, where $\pi$ : $U \rightarrow U / \Gamma$ is the projection mapping, then there are elliptic elements $\gamma_{1}$, $\gamma_{2} \in \Gamma, \gamma_{1}\left(z_{1}\right)=z_{1}, v_{1}=\operatorname{order}\left\langle\gamma_{1}\right\rangle=2 ; \gamma_{2}\left(z_{2}\right)=z_{2}, v_{2}=\operatorname{order}\left\langle\gamma_{2}\right\rangle=3$. Now $\operatorname{Mod}(\Gamma) \subsetneq$ Aut $T(\Gamma)$ because every quasi-conformal self-mapping $f$ of $U / \Gamma$ which leaves the set $\left\{p_{1}, p_{2}\right\}$ fixed induces an element of Aut $T(\Gamma)$, but only those $f$ which send every $p_{i}$ into a $p_{j}$ with $v_{i}=v_{j}, i, j=1,2$, correspond to elements in $\operatorname{Mod}(\Gamma) . \Omega=C-R$; then $T(\Gamma, \Omega)=T(\Gamma) \times T(\Gamma)$. Let $h \in$ Aut $T(\Gamma), h \notin \operatorname{Mod}(\Gamma)$. Then the mapping $h \times h \in$ Aut $T(\Gamma, \Omega)$, but $h \times h$ $\notin \operatorname{Mod}(G, \Omega) H$. The same argument applies unchanged for a whole collection of finitely generated Fuchsian groups $\Gamma$ with signature $(g, n$; $v_{1}, \ldots, v_{n}$ ) such that $g \geqslant 2, n \geqslant 2,2 \leqslant v_{i} \leqslant \infty$ and $v_{i}<v_{j}<\infty$ for at least two indices $i$ and $j$.

In the following theorem we drop the requirement that the components $\Delta_{i}$ of $G$ be simply connected.

THEOREM VIII. Let $G$ be a finitely generated nonelementary, nonexceptional Kleinian group. Assume that $G$ does not contain elliptic elements. Then Aut $T(G, \Omega)$ is the semidirect product of $\operatorname{Mod}(G, \Omega)$ by $K$, where $K=\{h \in$ Aut $T(G, \Omega) / h$ lifts to an $\tilde{h} \in H\}$ ( $H$ previously defined in $\S 3)$, if and only if the normalizer of $\operatorname{Mod}_{H_{i}}\left(\Gamma_{i}\right)$ in $\operatorname{Mod}\left(\Gamma_{i}\right)$ is $\operatorname{Mod}^{H_{i}}\left(\Gamma_{i}\right), 1 \leqslant i \leqslant n$.

REMARK. Since $\operatorname{Mod}_{H_{i}}\left(\Gamma_{i}\right)$ is a normal subgroup of $\operatorname{Mod}^{H_{i}}\left(\Gamma_{i}\right), \operatorname{Mod}^{H_{i}}\left(\Gamma_{i}\right)$ is contained in the normalizer of $\operatorname{Mod}_{H_{i}}\left(\Gamma_{i}\right)$ in $\operatorname{Mod}\left(\Gamma_{i}\right)$. 
Proof. We recall that $T\left(\Gamma_{1}\right) \times \cdots \times T\left(\Gamma_{n}\right)$ is the universal covering space of $T(G, \Omega)$ and that

$$
T(G, \Omega)=\frac{T\left(\Gamma_{1}\right) \times \cdots \times T\left(\Gamma_{n}\right)}{\operatorname{Mod}_{H_{1}}\left(\Gamma_{1}\right) \times \cdots \times \operatorname{Mod}_{H_{n}}\left(\Gamma_{n}\right)}
$$

(see [6], [7]). By a general covering space theory, it is not difficult to show that $f \in$ Aut $T(G, \Omega)$ if and only if a lifting $\tilde{f}$ belongs to the normalizer of $\operatorname{Mod}_{H_{1}}\left(\Gamma_{1}\right) \times \cdots \times \operatorname{Mod}_{H_{n}}\left(\Gamma_{n}\right)$ in Aut $T\left(\Gamma_{1}\right) \times \cdots \times T\left(\Gamma_{n}\right)$. From a previous theorem, Aut $T\left(\Gamma_{1}\right) \times \cdots \times T\left(\Gamma_{n}\right)$ is the semidirect product of $\operatorname{Mod}\left(\Gamma_{1}\right) \times \cdots \times \operatorname{Mod}\left(\Gamma_{n}\right)$ by $H$ (see $\S 3$ ). From another result (in this section), every $f \in$ Aut $T(G, \Omega)$ has the property that

$$
f_{\mid T\left(\Gamma_{i}\right) / \operatorname{Mod}_{H_{i}}\left(\Gamma_{i}\right)}: \frac{T\left(\Gamma_{i}\right)}{\operatorname{Mod}_{H_{i}}\left(\Gamma_{i}\right)} \stackrel{\text { onto }}{\rightarrow} \frac{T\left(\Gamma_{\sigma(i)}\right)}{\operatorname{Mod}_{H_{\sigma(i)}}\left(\Gamma_{\sigma(i)}\right)}
$$

biholomorphically for some $\sigma \in S_{n}$. Hence $f \in$ Aut $T(G, \Omega)$ and $\sigma=$ id, if and only if a lift of such an $f, \tilde{f}_{\mid T\left(\Gamma_{i}\right)}: T\left(\Gamma_{i}\right) \rightarrow T\left(\Gamma_{i}\right)$ belongs to the normalizer of $\operatorname{Mod}_{H_{i}}\left(\Gamma_{i}\right)$ in $\operatorname{Mod}\left(\Gamma_{i}\right)$, all $i$. $\operatorname{But} f \in \operatorname{Mod}(G, \Omega)$ if and only if the normalizer of $\operatorname{Mod}_{H_{i}}\left(\Gamma_{i}\right)$ in $\operatorname{Mod}\left(\Gamma_{i}\right)$ is $\operatorname{Mod}^{H_{i}}\left(\Gamma_{i}\right)$, all $i$. Now if $\sigma \neq \mathrm{id}$, we again lift $f$ to an $\tilde{f}$ and conclude that $\tilde{f} \in H$, hence by definition, $f \in K$. Once one realizes that all such $f \in$ Aut $T(G, \Omega), \sigma \neq$ id can arise in this fashion only, that is $\tilde{f} \in H$, the result is proven.

Proposition II. Let $G$ be a finitely generated nonelementary, nonexceptional Kleinian group. Then $T(G, \Omega)$ is not homogeneous, that is, if $x, y \in T(G, \Omega)$ are arbitrary, there does not exist in general an $h \in$ Aut $T(G, \Omega)$ such that $h(x)=y$.

Proof. We remark that if $x=\left(x_{1}, \ldots, x_{n}\right) \in T(G, \Omega)$ then (via the isomorphism theorem, §5), each $x_{i}$ is an equivalence class of points in $T\left(\Gamma_{i}\right)$, under $\operatorname{Mod}_{H_{i}}\left(\Gamma_{i}\right) \subseteq \operatorname{Mod}\left(\Gamma_{i}\right)$. Since $\operatorname{Mod}\left(\Gamma_{i}\right)$ identifies conformally equivalent Riemann surfaces (see [1]), any two Riemann surfaces representing $x_{i}$ are conformally equivalent. Let $x=\left(x_{1}, \ldots, x_{n}\right) \in T(G, \Omega)$ be arbitrarily chosen.

Choose $y=\left(y_{1}, \ldots, y_{n}\right) \in T(G, \Omega)$ such that a Riemann surface in the equivalence class $y_{1}$ is not conformally equivalent to a representative Riemann surface from any of the $x_{i}, 1 \leqslant i \leqslant n$ (this can always be done assuming the hypothesis). Suppose there existed an $h \in$ Aut $T(G, \Omega)$ such that $h(x)=y$. Lift $h$ to an $\tilde{h}$ on the universal covering space $T\left(\Gamma_{1}\right) \times \cdots \times$ $T\left(\Gamma_{n}\right)$. By a previous theorem (see $\S 3$ ), $\tilde{h}$ is an element of the semidirect product of $\operatorname{Mod}\left(\Gamma_{1}\right) \times \cdots \times \operatorname{Mod}\left(\Gamma_{n}\right)$ by $H$. Since each element of $\operatorname{Mod}\left(\Gamma_{i}\right)$, each $i$, identifies conformally equivalent Riemann surfaces [1], so does $\operatorname{Mod}\left(\Gamma_{1}\right) \times \cdots \times \operatorname{Mod}\left(\Gamma_{n}\right)$ on $T\left(\Gamma_{1}\right) \times \cdots \times T\left(\Gamma_{n}\right)$ componentwise. 
Similarly, any element of $H$ identifies conformally equivalent Riemann surfaces by looking at the induced mapping on the cotangent space, using the cotangent space splitting theorem (see §1), and applying Royden's result (and generalization, see [5]). Hence every element of Aut $T\left(\Gamma_{1}\right) \times \cdots \times T\left(\Gamma_{n}\right)$ identifies conformally equivalent Riemann surfaces. In particular, $\tilde{h} \in$ Aut $T\left(\Gamma_{1}\right) \times \cdots \times T\left(\Gamma_{n}\right)$. But $\tilde{h}$ maps some lift of $x$, via the covering mapping, denoted $\tilde{x}=\left(\tilde{x}_{1}, \ldots, \tilde{x}_{n}\right)$ to a lift of $y$, denoted $\tilde{y}=\left(\tilde{y}_{1}, \ldots, \tilde{y}_{n}\right)$. Then, in particular, a Riemann surface representing $\tilde{y}_{1}$ must be conformally equivalent to a Riemann surface representing $\tilde{x}_{i}$ for some $i$. However, by the opening remark and the way $y=\left(y_{1}, \ldots, y_{n}\right)$ was chosen, we arrive at a contradiction.

\section{REFERENCES}

1. L. Bers, Uniformization, moduli, and Kleinian groups, Bull. London Math. Soc. 4 (1972), 257-300.

2. ___ Fiber spaces over Teichmüller spaces, Acta Math. 180 (173), 89-126.

3. L. Bers and L. Greenberg, Isometries between Teichmüller spaces, Advances in the Theory of Riemann Surfaces, Ann. of Math. (2) 66 (1971), 53-79.

4. C. Earle and I. Kra, On isometries between Teichmüller spaces, Duke Math. J. 41 (1974), 583-591.

5. __ On holomorphic mappings between Teichmüller spaces, in Contributions to Analysis, Academic Press, New York, 1974, pp. 107-124.

6. I. Kra, Deformation spaces, crash course in Kleinian groups, Lecture Notes in Math., vol. 400, Springer-Verlag, Berlin, 1974, pp. 48-70.

7. B. Maskit, Self-maps of Kleinian groups, Amer. J. Math. 93 (1971), 840-856.

8. H. L. Royden, Automorphisms and isometries of Teichmüller space, Ann. of Math. Studies, no. 66, Princeton Univ. Press, Princeton, N. J., 1971, pp. 369-383.

9. _ Remarks on the Kobayashi metric, Lecture Notes in Math., vol. 185, SpringerVerlag, Berlin, 1971, pp. 125-137.

10. J. A. Gentilesco, Automorphisms of the deformation space of a Kleinian group, Doctoral Dissertation, SUNY, Stony Brook.

Department of Mathematics, State University of New York at Stony Brook, Stony BroOK, NEW YoRK 11794

Current address: Department of Mathematics, University of Pittsburgh, Pittsburgh, Pennsylvania 15261 